\title{
REVIEW
}

\section{The effect of light on critical illness}

\author{
Ricardo Castro*, Derek C Angus², Matt R Rosengart ${ }^{3}$ \\ This article is one of eleven reviews selected from the Annual Update in Intensive Care and Emergency Medicine 2011 (Springer Verlag) and \\ co-published as a series in Critical Care. Other articles in the series can be found online at http://ccforum.com/series/annual. Further \\ information about the Annual Update in Intensive Care and Emergency Medicine is available from http://www.springer.com/series/8901
}

\author{
"We shape our buildings, and afterwards \\ our buildings shape us."
}

Winston Churchill

\section{Introduction}

The research of this decade has yielded substantial improvements in the delivery of and technology with which to provide care for critically ill intensive care unit (ICU) patients. Garnering less attention from the medical and scientific community is the environment in which that care is provided, which remains impersonal, noisy, and over illuminated. Noticeably, the nursing and business literature is replete with studies on the matter $[1,2]$.

This discussion will focus on the available evidence regarding associations between the ICU environment, specifically light, and patient outcome. Definitions of light and the biology, including neural, hormonal, and immunologic mechanisms, by which it affects the body will be initially emphasized. An integrative commentary will be presented at the conclusion. Because of constraints, the focus is upon the critically ill patient, recognizing that much of what will be discussed is equally applicable to the healthcare provider.

\section{Light}

Sunlight reaching the earth's surface is categorized by effective wavelength: ultraviolet B (UV-B, 280-315 nm), ultraviolet A (UV-A, 315-400 nm), visible light (400$760 \mathrm{~nm})$, and infrared light $(760 \mathrm{~nm} \times 1.06 \mathrm{~nm})$ [3]. Of these four categories, visible light is essential for vision and resetting of the circadian clock through photoreceptors in the retina [4]. Exposure to UV-B radiation induces biological changes in the integument, such as sunburn, skin cancers and, as will be discussed, immunosuppression [5]. UV-A is involved in carcinogenesis

*Correspondence: castror@umpc.edu

'Department of Critical Care Medicine,University of Pittsburgh Medical Center, CRISMA Center, 605 Scaife Hall, 3550 Terrace Street, Pittsburgh, PA 15261, USA Full list of author information is available at the end of the article through the generation of highly reactive chemical intermediates and lipid peroxidation [6].

Light is measured using either radiometry (an analysis of the entire visible and non-visible wavelength spectra) or photometry [7]. Both methods provide valuable and distinct information that defines light. Photometry, a perception of brightness as seen by the human eye, is performed with a lux meter in units called lux. For comparison purposes, moonlight is 0.5 to 1 lux, a bright office is 400 lux, and a sunny day in spring is 32,000 to 60,000 lux [8]. Nocturnal light levels vary among ICUs with mean maximum levels ranging from 1 to 1,400 lux [8]. During the performance of procedures (e.g., catheter insertion), light devices can easily deliver > 10,000 lux.

Light affects the body by receptor stimulation through the eyes (retina) and through the skin. The classical visual sensory system is comprised of photoreceptor cells of rods (low-level light) and cones (sharpness, detail, and color vision). The impact of a photon of light generates rhodopsin, thus creating electrical impulses in the optical nerve that converge within the visual cortex and are interpreted as 'vision' [4]. For more than 150 years, scientists considered rods and cones to be the sole photoreceptor cells in the eye. With the discovery of a novel, third type of retinal photoreceptor in mammals [9], a new retinohypothalamic pathway was described, providing evidence of a pathway mediating the biological but non-visual effects of light.

\section{The biological perspective: non-visual effects of light}

The health effects of light are realized through several biological processes additional to and independent of the ability of visually perceiving the external world [10]. Only recently have we acquired deeper insight into the biological mechanisms regulating these non-visual effects. Fundamental to this understanding is an appreciation of how light controls the biological clock and regulates important hormones through seasonal photoperiods (duration of an organism's daily exposure to light) and regular light-darkness rhythms. 


\section{Circadian pathways}

Circadian rhythms are cycles of physiologic processes and behaviors driven by an endogenous oscillator having a period of approximately ( $\operatorname{circa}$ ) one day (diem). The most evident circadian rhythm in humans is the sleepwake cycle. Other circadian rhythms include body temperature, release of hormones (e.g., melatonin, cortisol), and gene expression. These rhythms persist with a near 24-h period even in the absence of time-of-day information [11]. Environmental stimuli can reset the phase of the circadian pacemaker, light being the ultimate entrainment signal [12]. A change in the timing of the light-dark cycle (e.g., nocturnal light exposure) will result in a shift in the phase of circadian rhythms that can only be detected in the next circadian cycle. However, the effects on circadian physiology (e.g., body temperature and melatonin suppression) can be observed during or immediately after the light exposure [13]. In the case of a disruption of the rhythm, exposure to bright light in the morning will help to restore it [14].

The suprachiasmatic nucleus in the anterior hypothalamus is the circadian pacemaker [15]. It contains cells that are able to express sustained periodicity, even in vitro. Functional neuroimaging studies have demonstrated that light quickly activates alertness-related subcortical structures in the suprachiasmatic nucleus and a sequence of intermediate connections terminating in the pineal gland that underlie the circadian-based synthesis and release of melatonin [16]. The thalamus functions as an interface between alertness, cognition, and the effects of light [17], anatomically connecting with the frontal, temporal and cerebral cortex (except for the olfactory system), cerebellum, and basal ganglia. It regulates the flow of information from the retina to the visual cortex or between cortical areas [18]. Light stimulates a retinal photoreceptor system expressing melanopsin, a photopigment produced in the human inner retina and directly activated by light [4]. Interestingly, even extensive degradation of the photoreceptor apparatus does not eliminate the synthesis of melanopsin [10]. Subsequent signals are channeled to the suprachiasmatic nucleus via the retinohypothalamic pathway. Melanopsin plays a key role in mediating the non-visual effects of light and renders a small subset of retinal ganglion cells intrinsically photosensitive (ipRGC) with maximal sensitivity to blue light [11]. The efferent projections of the ipRGCs include multiple hypothalamic, thalamic, striatal, brainstem and limbic structures, which govern circadian cycles, body temperature, and alertness [17].

The ability of light to modulate cortical activity and circadian rhythm is defined, in part, by the duration, intensity and wavelength of the lighted stimulus $[17,19]$. Biological processes dictate that non-visual responses are maximally sensitive to blue light $(459-483 \mathrm{~nm})$, in contrast to the green $(\sim 550 \mathrm{~nm})$ spectral sensitivity of classical visual photoreceptors [11,13]. Blue light most powerfully changes the rhythm of melatonin and cortisol secretion, acutely suppressing melatonin. It also elevates body temperature and heart rate, reduces subjective sleepiness and improves alertness [17,20,21]. In one study, office workers were exposed to two new lighting conditions for 4 weeks: A blue-enriched white light or a white light that did not compromise visual performance. Blue-enriched white light significantly heightened subjective measures of alertness, positive mood, performance, and concentration while reducing evening fatigue, irritability, and eye discomfort. Daytime sleepiness was reduced and the quality of subjective nocturnal sleep was improved [21]. Thus, evidence confirms that for the human brain, the absence of blue light, at least from a circadian point of view, is effectively darkness [22].

\section{Melatonin}

Most of the effects of the photoperiod are mediated by melatonin, the hormone secreted by the pineal gland in response to darkness. This hormone is synthesized within the pineal gland from the essential amino acid tryptophan through enzymatic processes of 5-hydroxylation and decarboxylation that yield 5-hydroxytryptamine (5-HT or serotonin). During daylight, serotonin remains stored in pinealocytes and unavailable for conversion to melatonin. With darkness, postganglionic sympathetic outflow to the pineal gland releases serotonin and induces enzymatic conversion of serotonin to melatonin [23].

Melatonin plays an equally important role in the adaptive response of an organism to environmental challenges. Experimental studies have shown that binding of melatonin to specific receptors in antigen-activated Type 1 T-helper cells (Th-1) upregulates pro-inflammatory cytokine production (such as interferon [IFN]-X and interleukin [IL]-2) [24] and enhances the production of IL-1, IL-6 and IL-12 in human monocytes [25-27]. It is believed that it may increase phagocytosis and antigen presentation [28]. Animal models have demonstrated that melatonin has a protective effect in mice against lethal viral encephalitis [29], infectious hepatitis [30], and hemorrhagic [31] or septic [32] shock. In this context, melatonin has been shown to prevent endotoxin-induced circulatory failure in rats through inhibition of tumor necrosis factor (TNF)- $\alpha$, and to reduce post-shock levels of IL-6, superoxide production in the aorta, and inducible nitric oxide synthase (iNOS) in the liver [32] (Table 1).

These data suggest that the winter immunoenhancement paradigm [38] could explain photoimmunomodulatory processes in animals and be applicable to patients contending with severe illnesses. This theory was developed in the context of lower mammals and proposes that in environments that undergo seasonal changes in 


\section{Table 1. Examples of immune effects associated with photoperiods}

Tumorigenesis was reduced and basal lymphocyte proliferation or mitogen-induced splenocyte proliferation were promoted with shorter days (rodents) [33, 34] Seasonal attenuation of the immune response to Gram-negative infections was observed when shortening the length of days in a rodent model [35]

Measures of immune cell counts, lymphoid organ weights or T cell-dependent antibody responses to xenogeneic antigens were generally enhanced by short photoperiod of winter [36]

Exposure to short days increased mass of the spleen and enhanced the total number of leukocytes and lymphocytes when only photoperiod was manipulated [20] Circulating numbers of leukocytes, neutrophils, and lymphocyte proliferation in response to mitogens were higher in winter than in the summer in a primate model [37]

Seasonal changes in immune parameters were observed, with enhancement of specific immune responses during autumn and winter compared with spring and summer, in animal models (rodents, rabbits, dogs and primates) [20]

energy availability, selection should favor individuals that support enhanced immune function during the winter (shorter days). Photoperiodic information is used to bolster immune function in anticipation of winter [38]. Redirecting metabolic energy stores toward improved immune function should enable animals to contend better with the stressors (e.g., decreased temperature and food availability) of winter, a time of the year when reproductive efforts are less likely to succeed. Conversely, during the breeding season (longer days), energetic tradeoffs favor reproduction, and immune function is relatively impaired [34].

A critically ill patient lies in a winter-like condition because energy resources are severely compromised. Moreover, immunity is impaired as the body is contending with many severe insults. The physiological regulation of melatonin secretion by darkness and light is probably abolished due to loss of the circadian rhythm, a consequence of the altered patterns of illumination in most ICUs [39]. Thus, this pathway is directly linked to the inflammatory response and, ultimately, a patient's outcome. It would be highly desirable to direct resources toward enhancing the immune system so as to enable the patient with a better chance to overcome this biological 'severe weather'. This might be accomplished by restoring a circadian light/darkness cycle, by providing longer periods of darkness and less hours of light in the ICU. The use of 'virtual darkness' by providing amber lenses to filter the impact of electrical light, particularly ubiquitous blue light, could attain the objective [22]. Beyond its antioxidant properties, the role of melatonin as a systemic immunoregulatory agent sensitive to exogenous regulation is an exciting idea to be tested in controlled trials of human sepsis [40].

\section{Cortisol}

Cortisol is a steroid hormone that influences metabolic, immunologic, muscle and brain functions. Its secretion is regulated primarily by the hypothalamic-pituitaryadrenal (HPA) axis through release of corticotrophin releasing hormone $(\mathrm{CRH})$ from the hypothalamus and adrenocorticotrophic hormone (ACTH) from the anterior pituitary gland [41]. Cortisol negatively feeds back to the hippocampus, hypothalamus, and the anterior pituitary, inhibiting $\mathrm{CRH}$ and $\mathrm{ACTH}$. The suprachiasmatic nucleus regulates the circadian rhythm of corticosteroids [42]. Thus, cortisol decreases across the habitual waking day to attain a nadir near bedtime. Concentrations subsequently increase during the darkness of night and peak near arousal, regardless of continuous wakefulness or sleep [43]. Superimposed on this rhythm are fluctuations associated with the pulsatile or acute release of cortisol by diverse factors such as anxiety, stress, immune challenge, blood glucose levels, sleep onset, sleep loss, and exposure to light [44].

In sepsis, the HPA axis affects inflammation by modulating leukocytes, cytokines and NO synthesis [45]. Through negative feedback, inflammatory cytokines may suppress sensitivity to ACTH [46], resulting in adrenal insufficiency [47], or compete with intracellular glucocorticoid receptor function, thereby causing peripheral tissue glucocorticoid resistance [48].

The relationship between light and plasma levels of cortisol is complex. Inconsistent results have been attributed to differences in light intensity and wavelength, and the timing of application as it relates to the circadian cycle [44]. More recent studies, however, provide compelling evidence that light is a strong determinant of cortisol concentration. Bright light exposure (up to 10,0000 lux) elicited a significant suppressive effect when applied either on the rise or descent phase of cortisol rhythm. Lower intensities (less than $\sim 5,000$ lux) failed to induce significant changes [44]. These results would be consistent with the findings of light-intensity response curves for melatonin suppression [49]. In contrast to melatonin's responses, both blue and red lights increased cortisol plasma levels at night [50].

A multisynaptic neural pathway (retina-suprachiasmatic nucleus-adrenal gland) that bypasses the HPA axis is considered responsible for the acute influence of light on corticosteroid concentrations. These conclusions stem, in part, from the observation that cortisol variations are reported to be dependent upon an intact suprachiasmatic nucleus and not related to changes in 
ACTH levels [51]. Thus, aspects of a lighted environment could be adjusted to elicit this HPA-independent response. In a critically ill patient, this approach could lessen a relative or overt adrenal insufficiency and constitutes an interesting idea worthy of future study.

\section{Photo-immunomodulation}

Seasonal rhythms and fluctuations in innate and acquired immune responses have been documented in many species [52,53]. Profound but selective effects on immune function are associated with the prevailing photoperiod $[36,54]$. T cell immunity is depressed in most species in the winter, even when natural light sources and exposure are kept constant $[20,54]$. Experimental data, however, show that immune cell numbers and immunoglobulin concentrations vary with respect to the season or day length $[34,54]$ even during the winter. Higher leukocyte counts are noted with less hours of light [20,54], demonstrating that the photoperiod may also influence the functional capabilities of immune cells. Short days selectively enhance natural killer (NK) basal proliferative capacity and cell activity [34]. In contrast, in the same rodent model, phagocytic and granulocyte oxidative burst activity are reduced during short, by comparison to long, days [20,55]. Collectively, these results confirm reduced immune function in winter compared to summer, but with enhanced immune function in short winter-like photoperiods compared to long summer-like day lengths [56] (Table 1). The net elevated immune function in short days is thought to counteract the suppressive effects of environmental stressors such as low ambient temperature on immune function [20]. These facts raise many questions for the management of critically ill patients. Is there a consistent seasonality on the outcomes of critically ill patients? Should we shorten the day length for the most seriously ill septic patients in the ICU to enhance their immunity? These concepts await further investigation.

\section{Central pathways: the inflammatory reflex}

A recent major advance in our understanding of the immune response during severe sepsis came with the identification of the cholinergic anti-inflammatory pathway [57]. Cytokine release can be controlled at multiple levels, including the central nervous system (CNS). Endotoxin and products of inflammation stimulate afferent neural signals in the vagus nerve that induce acutephase responses, fever, and the upregulation of IL- $1 \beta$ in the brain. Concomitantly, afferent vagus nerve signals are transmitted to the medullary reticular formation, locus ceruleus, hypothalamus, and dorsal vagal complex, leading to an increase in ACTH from the anterior pituitary gland [57]. This stimulates an increase in systemic glucocorticoid levels, thereby inhibiting pro-inflammatory cytokine release [58]. Alternatively, ascending sensory fibers of the vagus nerve that synapse in the nucleus tractus solitarius of the upper medulla can inhibit cytokine release. Like other reflex arcs, the inflammatory reflex is comprised of a sensory afferent arm (described above) and an efferent motor arm that controls a rapid and opposing reaction [57]. This cholinergic anti-inflammatory efferent pathway inhibits inflammation. Efferent vagus nerve signals release acetylcholine $(\mathrm{ACh})$ in organs of the reticuloendothelial system, including the spleen, liver, and gastrointestinal tract [57]. ACh binds to the nicotinic receptor $(\alpha 7 \mathrm{nAChR})$ expressed on the surface of activated macrophages and other immune cells, which inhibits nuclear factor $\kappa B(\mathrm{NF}-\mathrm{\kappa B})$ and attenuates cytokine production. The biological relevance of this pathway was made manifest by murine endotoxemia studies demonstrating that stimulation of the efferent vagus nerve inhibited TNF- $\alpha$ release, prevented shock, and improved survival [59]. The vagal inflammatory reflex also regulates localized inflammation. In a murine model of arthritis, vagus nerve stimulation inhibited inflammation and suppressed the development of paw swelling [60]. In the lungs, pharmacological $\alpha 7 n A C h R$ stimulation correlated with reduced lipopolysaccharide (LPS)-induced neutrophil recruitment [61]. Collectively, these studies suggest that either by electrical or chemical intervention, this inflammatory reflex pathway can be modified to modulate the inflammatory response to injury or infection [62].

Consistent evidence supporting a link between sunlight exposure and the inflammatory reflex is lacking, however. The efferent arm of the inflammatory reflex regulates TNF- $\alpha$ production in the spleen via two serially connected neurons: One preganglionic, originating in the dorsal motor nucleus of the vagus nerve (parasympathetic), and the second postganglionic, originating in the celiac-superior mesenteric plexus, and projecting in the catecholaminergic splenic (sympathetic) nerve [63]. Therefore, one of the most crucial components of the efferent inflammatory reflex is catecholaminergic in nature. As the suprachiasmatic nucleus balances sympathetic and parasympathetic output to peripheral organs [64], one might speculate that the efferent arm of the inflammatory reflex could be directly activated or inhibited by light exposure, thereby establishing a neural link between the retinohypothalamic pathway and the inflammatory reflex. As the non-visual retinohypothalamic pathway's net effect is to enhance immunity, this inflammatory reflex mechanism could constitute a counterregulatory mechanism (Fig. 1).

Skin pathways: immunosuppression by ultraviolet B radiation The skin represents an important interface between the external environment and internal tissues and is 


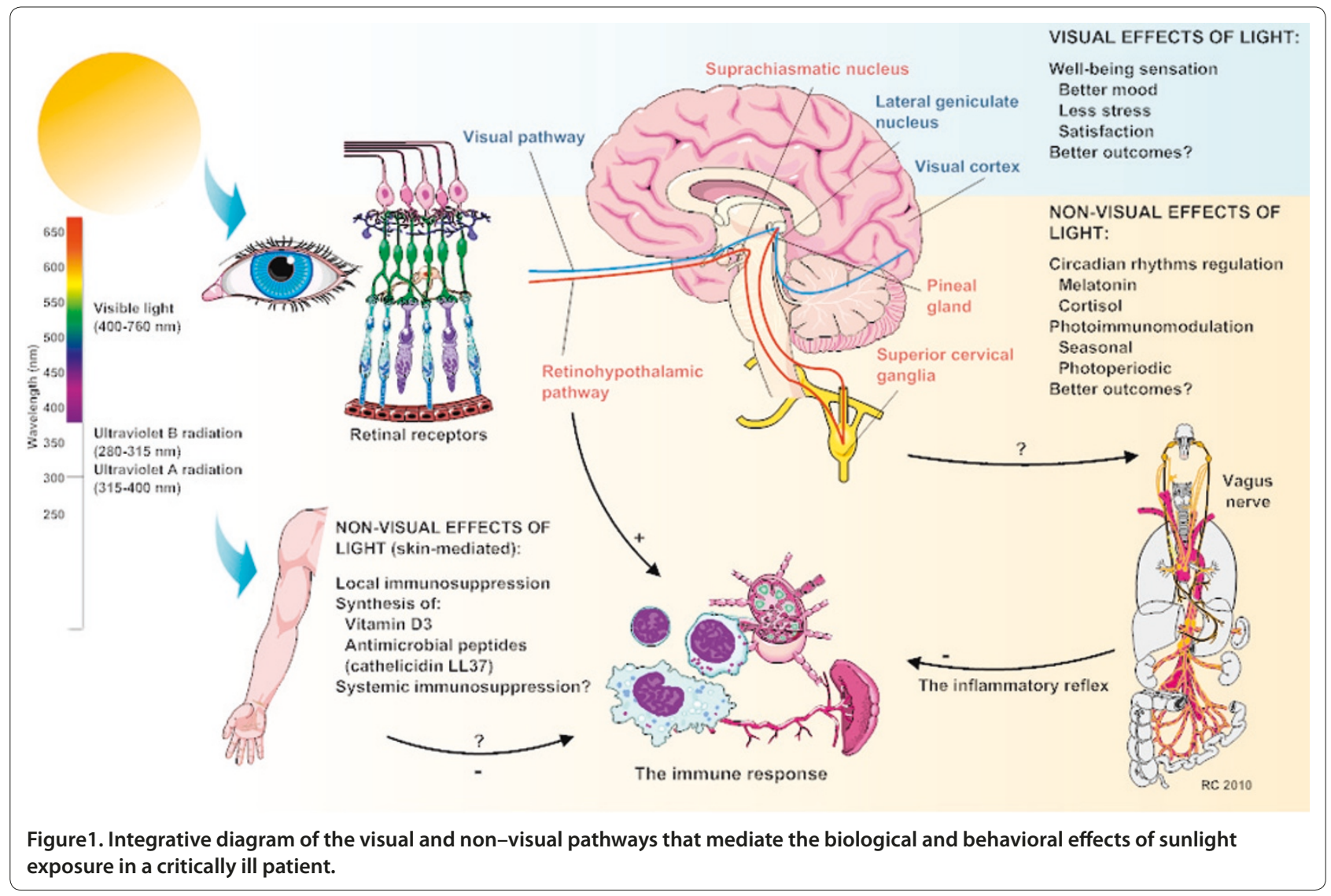

constantly bathed in sunlight. Both direct (skinmediated) and indirect immunomodulation have been described. Visible light (400-700 nm) can penetrate the epidermal and dermal layers and directly interact with circulating lymphocytes. UV-B and UV-A radiation alter normal human immune function predominantly via a skin-mediated response [20]. Epidermal Langerhans cells survey invading agents and transmit the information into immune cells. After engulfing exogenous antigen, these sentinels migrate to draining lymph nodes and present the processed antigen to $T$ cells, thereby inducing specific $\mathrm{T}$ cell differentiation and $\mathrm{T}$ cell activation. Ionizing and non-ionizing UV radiation (below $400 \mathrm{~nm}$ ) inhibit this antigen presentation via induction of suppressive keratinocyte-derived cytokines. This reduces effector $\mathrm{T}$ cell proliferation and activity and induces immunotolerance [65]. In addition, regulatory $\mathrm{T}$ cells (Treg) serve important immunoregulatory and immunosuppressive functions. Induced by UV radiation, Treg cells release IL-10, leading to immunosuppression. Thus, functional alterations of epidermal Langerhans cells and a systemic increase in Treg cells couple the epidermis to local and systemic immunosuppression [66]. The balance between the numbers and function of regulatory and effector $\mathrm{T}$ cells is crucial for the immune system. Although the molecular mechanisms underlying the expansion of regulatory T cells after UV exposure are largely unknown, vitamin D3 has been recently shown to upregulate the RANKL (receptor activator for NF-kB ligand) expression that activates Langerhans cells [65]. This should be carefully considered when managing critically ill patients in an ICU with windows with no UV protection. Although not subjected to rigorous evaluation, UVinduced immunosuppression could play an adverse role in a critically ill patient (Fig. 1).

\section{Vitamin D3, 1,25(OH)D2, and cathelicidin}

Vitamin D belongs to the family of steroid hormones. Exposure to UV-B radiation of 290-315 nm converts 7-dehydrocholesterol to pre-vitamin D3. Pre-vitamin D rapidly undergoes a thermally induced isomerization to form vitamin D3. D3 enters the circulation where it undergoes hydroxylation in the liver by vitamin D-25hydroxylase and in the kidney by the 25-hydroxyvitamin D-1-alpha-hydroxylase ( $1 \alpha$-OHase), thus forming $1-$ $25(\mathrm{OH}) \mathrm{D} 2$. The classic function of vitamin $\mathrm{D}$ is to enhance intestinal absorption of calcium by regulating several calcium transport proteins in the small intestine [67].

Cells of the immune system also possess $1 \alpha-\mathrm{OHase}$ and the vitamin $\mathrm{D}$ receptor (VDR) and, thus, are able to 
Table 2. Some beneficial health effects of light exposure reported in the literature

Light can alleviate seasonal depression [74]

Sunlight exposure improves cognitive function among depressed people in a dose-response relationship [75]

Light regulates melatonin, which has paramount immunomodulatory effects and has been shown to reduce breast cancer growth [20]

Female patients with a first cardiac attack treated in sunny rooms had a shorter stay than female patients treated in dull rooms and mortality in both sexes was consistently higher in dull rooms than in sunny rooms [76].

Absence of visible daylight in the room is significantly associated with delirium and higher risk of dementia in intensive care patients [77]

produce the hormonally active form. Macrophages produce the antimicrobial peptide, cathelicidin LL-37, in response to endogenously produced $1,25(\mathrm{OH}) \mathrm{D} 2$ to enhance innate immunity [67]. The antimicrobial peptide, LL-37, is the only known member of the cathelicidin family expressed in humans. It is a multifunctional host defense molecule essential for normal immune responses to infection and tissue injury. LL-37 peptide exhibits strong activity against common ICU bacterial strains, including Escherichia Coli, Pseudomonas aeruginosa, Klebsiella pneumoniae, Staphylococcus aureus (methicillinresistant [MRSA] and non-MRSA), and Neisseria gonorrhoeae. It prevents the immunostimulatory effects of bacterial cell wall molecules such as LPS and can, therefore, protect against lethal endotoxemia [68]. Cellular production of LL-37 is affected by multiple factors, including bacterial products, host cytokines, availability of oxygen, and sun exposure through the activation of CAP-18 gene expression by vitamin D3 [68]. As sunlight within the UV-B spectrum induces immunosuppression and heightens vulnerability to infection, 1,25(OH)D2 potentially balances this effect by stimulating the synthesis of LL-37 in the skin and circulating phagocytic cells [69]. Recently, lower circulating levels of 25(OH)D and vitamin $\mathrm{D}$ binding protein have been observed in critically ill patients compared to healthy controls [70]. Thus, it might be concluded that optimal function of our innate immune system requires some necessary amount of vitamin D and, accordingly, of sunlight (Fig. 1). This is a strong reason for providing septic patients with controlled exposure to direct sunlight.

\section{The biological perspective: visual effects of light}

From the Greek Asclepieia to the monastic Middle Age infirmaries, traditions of complementary medicine and holistic healing have been rooted in the provision of medical care. Pleasant views were obligatory characteristics of places designed to give shelter and provide care for diseased people. It is now appreciated that the visual environment can powerfully influence the atmosphere and visual impression of the workplace. Properly designed, the overall working environment can have a stimulating effect on the people working within it [71]. Interior daylight contributes substantially to the perceived quality of the working environment. Light is mood enhancing and fosters visual and general health [71]. An important benchmark in the history of integrating nature into the care of patients was made by Roger Ulrich in 1984 [72]. Post-surgical patients with a view of nature suffered fewer complications, used less pain medication, and were discharged sooner than those with a view of a brick wall. This pioneering study provided the first formal scientific evidence that 'healing environments' beneficially alter health. In the following years, many other groups from across the world have reported the health benefits associated with views of nature, daylight exposure and related elements [73] (Table 2). Based on these findings, many have proposed that exposure to daylight be considered as a medical intervention for critical care patients. Nevertheless, such studies have not been yet performed though the concept warrants further study.

\section{The behavioral perspective}

People prefer daylight to electric lighting as their primary source of illumination [78]. Most prefer to work and live in buildings illuminated by daylight as it provides psychological comfort, increased satisfaction in the work environment, and visual and general health [79]. A window providing a beautiful view of the surrounding landscape or of the sky and mountains might bolster psychological coping and thereby facilitate healing [71], all through a sensation of well-being. Well-being can be defined in terms of an individual's physical, mental, social, and environmental status. These aspects interact with each other and possess differing levels of importance specific to that individual (Table 3). Almost all of these components are present in the critically ill patient.

Apart from the biological considerations previously discussed, the positive sensations elicited by a daylighted view might enable a patient to more appropriately cope with critical illness. Psychologists make an important distinction between short-term positive emotions (hedonic well-being) and psychological (eudaimonic) well-being. Eudaimonic well-being has to do with the realization of personal potential and purpose in life, and is mainly determined by childhood social circumstances and the development of loving and trusting relationships early in life [81]. Therefore, it is not subject to simple modifications through daily life experiences. Conversely, hedonic 


\section{Table 3. Components of well-being [80]}

Individual characteristics of people such as functional ability and physical and mental health

Physical environmental factors including facilities, amenities, and housing standards

Social factors such as family and social networks

Living environment including household status, household conditions, and neighborhood

Socioeconomic factors including income, standard of living, and ethnicity

Personal autonomy factors such as ability to make choices and control

Subjective satisfaction on the person's evaluation of their quality of life

Psychological health such as psychological well-being, morale, and happiness

Activities such as hobbies, leisure, and social participation

Life changes such as traumatic or disruptive events or lack of change

Care including expectations, amount, and kind of support

well-being is related to experiences of happiness and satisfaction and is a short-term sensation. Several authors have described the short-term benefits of positive emotions and attitudes on reducing the cardiovascular response to stress [82], lowering pain ratings and sensitivity [83], and volunteers trained in meditation produced high levels of immunity to influenza [84]. Thus, the appreciation of sunlight may impact favorably upon the health of a critical patient through this shorter-term perspective (Fig. 1).

\section{The holistic perspective}

No single factor is responsible for any given health circumstance or condition. This common-sense statement was conceptually developed by Moos in 1976 [85] and is called the social-ecological framework. This model views a specific situation as the sum product of the interaction of many factors ordered in five levels: Individual, interpersonal, community-level, societal, and policy. Environment integrates into the third and fourth categories.

Humans can modify almost every aspect of their world to create hospitable places within which to work, play and live. They enjoy and seek the pleasant emotions that a beautiful landscape and a warm sunlight nourish. Over time, however, we have become extremely dependent upon a man-made environment. Artificial light constitutes an indispensable part of our modern lives. Consequently, seasons, daylight hours and healthy sleep-waking cycles are less a part of our existence. But physiology reminds us that maintaining a balanced sleep-wake cycle is essential to survive. It allows animals to enhance their immunity through light-mediated mechanisms even in adverse environmental conditions.

When a healthy individual suffers an acute serious illness, these ancient survival mechanisms reacquire relevance. The biologic environment becomes hostile and the patient starts to struggle with the most atavistic challenge he/she could face: The fight for survival. At this point, the provision of professional intensive care must include elements apart from standard medical care. It should consider the deliberate intention to modulate the patient's immune response via activation of visual and non-visual pathways. Modification of light settings and timing becomes a fundamental component in this approach, as well as prudent exposure to sunlight for some hours. We cannot assure that providing sunlight exposure to critically ill patients and shortening the daily time of exposition to light will result in improved survival. The final outcome will emerge from a dynamic ongoing process in which personal and environmental factors will exert influence upon each other according to the social-ecological framework. However, the systemic and local immunomodulatory effects and the positive emotions elicited by this sensorial experience give us a solid rationale to integrate them as key components in the delivery of care in the ICU.

\section{Conclusion}

Clearly light has the very real potential to alter the course of disease and the behavior of persons providing care. Although we have a deeper understanding of the biological mechanisms involved in the visual and non-visual effects of light, and the psychological and behavioral elements of the complex interaction between light exposure and health outcomes, it is far from complete. There are still many nebulous aspects, and with each step of understanding, several new questions arise, particularly in the context of critical illness. How does illness alter the neural and endocrine pathways governing the biological effects of light? Do measures to engage the physiologic and neural feedback loops enhance, hinder, or fail to influence their actions? What are the effects of blue and green light wavelengths in a patient that is sedated and intubated? What happens to the biologic rhythms and immune responses if our critically ill patient rests in a room without windows, even though it is a greatly illuminated one? As artificial light sources in ICUs fail to account for retinal spectral sensitivity and the circadian clock, are our artificially lighted work environments leaving our patients and healthcare providers blue light 'deprived'? Hopefully, for these and many other questions, future studies will enlighten us as to the benefits of returning natural light and nature to the bedside.

\section{Competing interests}

The authors declare that they have no competing interests.

\section{List of abbreviations used}

5-HT: 5-hydroxytryptamine; ACh: acetylcholine; ACTH: adrenocorticotrophic hormone; CNS: central nervous system; $\mathrm{CRH}$ : corticotrophin releasing hormone; HPA: hypothalamic-pituitary-adrenal; ICU: intensive care unit; 
IFN: interferon; IL: interleukin; iNOS: inducible nitric oxide synthase; LPS: lipopolysaccharide; NF-kB: nuclear factor KB; Th-1: Type 1 T-helper cells; TNF: tumor necrosis factor; Treg: regulatory $T$ cells.

\section{Author details}

'Department of Critical Care Medicine,University of Pittsburgh Medical Center, CRISMA Center, 605 Scaife Hall, 3550 Terrace Street, Pittsburgh, PA 15261, USA. ${ }^{2}$ Department of Critical Care Medicine, University of Pittsburgh, 614 Scaife Hall, 3550 Terrace Street, Pittsburgh, PA 15261, USA. ${ }^{3}$ Department of Trauma/General Surgery, UPMC - Presbyteria Hospital, F1266 Lothrop Street, Pittsburgh, PA 15213, USA.

\section{Published: 22 March 2011}

\section{References}

1. Stichler JF: Creating healing environments in critical care units. Crit Care Nurs Q 2001, 24:1-20.

2. Berry LL, Parker D, Coile RC Jr, Hamilton DK, O'Neill DD, Sadler BL: The business case for better buildings. Healthc Financ Manage 2004, 58:76-84.

3. Commission International de l'Éclairage: International Lighting Vocabulary, 4th edition. Paris: CIE; 1987.

4. Barrett KE, Barman SM, Boitano S, Brooks H: Vision. In Ganong's Review of Medical Physiology, 23rd edition. Edited by Barrett KE, Barman SM, Boitano S, Brooks H. New York: Mc-Graw Hill Companies; 2010:181-202.

5. Soter NA: Acute effects of ultraviolet radiation on the skin. Sem Dermatol 1990, 9:11-15.

6. Nishigori C: Cellular aspects of photocarcinogenesis. Photochemical and Photobiological Sciences 2006, 5:208-214.

7. Patel M Chipman J, Carlin BW, Shade D: Sleep in the intensive care unit setting. Crit Care Nurs Q 2008, 31:309-318.

8. Sabra Al: Theories of Light, from Descartes to Newton. New York: Cambridge University Press; 1981.

9. Brainard G, Hanifin J, Greeson J, et al:: Action spectrum for melatonin regulation in humans: evidence for a novel circadian photoreceptor. J Neurosci 2001, 21:6405-6412.

10. Terman $\mathrm{M}$, Terman J: A circadian pacemaker for visual sensitivity? Ann N Y Acad Sci 1985, 453:147-161.

11. Hankins MW, Peirson SN, Foster RG: Melanopsin: an exciting photopigment. Trends Neurosci 2008, 31:27-36.

12. Lack $L$, Wright $H$ : The effect of evening bright light in delaying the circadian rhythms and lengthening the sleep of early morning awakening insomniacs. Sleep 1993, 16:436-443.

13. Brainard GC, Hanifin JP: Photons, clocks, and consciousness. J Biol Rhythms 2005, 20:314-325

14. Bechtold DA, Gibbs JE, Loudon ASI: Circadian dysfunction in disease. Trends Pharmacol Sci 2010, 31:191-198.

15. Saper CB, Lu J, Chou TC, Gooley J: The hypothalamic integrator for circadian rhythms. Trends Neurosci 2005, 28:152-157.

16. Dijk D-J, von Schantz M: Timing and consolidation of human sleep, wakefulness, and performance by a symphony of oscillators. J Biol Rhythms 2005, 20:279-290.

17. Vandewalle G, Maquet P, Dijk DJ: Light as a modulator of cognitive brain function. Trends Cogn Sci 2009, 13:429-438.

18. Shipp S: The brain circuitry of attention. Trends Cogn Sci 2004, 8:223-230

19. Rea MS, Figueiro MG, Bierman A, Bullough JD: Circadian light. J Circadian Rhythms 2010, 8:2-12.

20. Haldar C, Ahmad R: Photoimmunomodulation and melatonin. J Photochem Photobiol B. 2010, 98:107-117.

21. Viola AU, James LM, Schlangen $L$, Dijk DJ: Blue-enriched white light in the workplace improves self-reported alertness, performance and sleep quality. Scand J Work Environ Health 2008, 34:297-306.

22. Phelps J: Dark therapy for bipolar disorder using amber lenses for blue light blockade. Med Hypotheses 2008, 70:224-229.

23. Wurtman RJ, Axelrod J, Phillips LS: Melatonin synthesis in the pineal gland: Control by light. Science 1963, 142:1071-1073.

24. Garcia-Maurino S, Gonzalez-Haba MG, Calvo JR, et al: Melatonin enhances IL-2, IL- 6, and IFN-gamma production by human circulating CD4+ cells: a possible nuclear receptor-mediated mechanism involving $\mathrm{T}$ helper type 1 lymphocytes and monocytes. J Immunol 1997, 159:574-581.

25. Garcia-Maurino S, Gonzalez-Haba MG, Calvo JR, Goberna R, Guerrero JM: Involvement of nuclear binding sites for melatonin in the regulation of
IL-2 and IL-6 production by human blood mononuclear cells. J Neuroimmunol 1998, 92:76-84.

26. Maestroni GJ: Therapeutic potential of melatonin in immunodeficiency states, viral diseases, and cancer. Adv Exp Med Biol 1999, 467:217-226.

27. Cutolo M, Maestroni GJ: The melatonin-cytokine connection in rheumatoid arthritis. Ann Rheum Dis 2005, 64:1109-1111.

28. Maestroni GJ: The immunotherapeutic potential of melatonin. Expert Opin Investig Drugs 2001, 10:467-476.

29. Ben-Nathan D, Maestroni GJ, Lustig S, Conti A: Protective effects of melatonin in mice infected with encephalitis viruses. Arch Virol 1995, 140:223-230.

30. Wang $H$, Wei W, Shen $Y X$, et al.: Protective effect of melatonin against liver injury in mice induced by Bacillus Calmette-Guerin plus lipopolysaccharide. World J Gastroenterol 2004, 10:2690-2696.

31. Wichmann MW, Haisken JM, Ayala A, Chaudry IH: Melatonin administration following hemorrhagic shock decreases mortality from subsequent septic challenge. J Surg Res 1996, 65:109-114.

32. Wu CC, Chiao CW, Hsiao G, Chen A, Yen MH: Melatonin prevents endotoxininduced circulatory failure in rats. J Pineal Res 2001, 30:147-156.

33. Hotchkiss AK, Nelson RJ: Melatonin and immune function: hype or hypothesis? Crit Rev Immunol 2002, 22:351-371.

34. Nelson RJ: Seasonal Patterns of Stress, Immune Function, and Disease. New York: Cambridge University Press, New York; 2002.

35. Baillie SR, Prendergast BJ: Photoperiodic Regulation of Behavioral Responses to Bacterial and Viral Mimetics: A Test of the Winter Immunoenhancement Hypothesis. J Biol Rhythms 2008, 23:81-90.

36. Nelson RJ: Seasonal immune function and sickness responses. Trends Immunol 2004, 25:187-192.

37. Mann DR, Akinbami MA, Lunn SF, Fraser HM, Gould KG, Ansari AA: Endocrineimmune interaction: alteractions in immune function resulting from neonatal treatment with a GnRH antagonist and seasonality in male primates. Am J Reprod Immunol 2000, 44:30-40.

38. Nelson RJ, Demas GE: Seasonal changes in immune function. Q Rev Biol 1996, 71:511-548.

39. Perras $B$, Meier $M$, Dodt $C$ : Light and darkness fail to regulate melatonin release in critically ill humans. Intensive Care Med 2007, 33:1954-1958.

40. Srinivasan V, Pandi-Perumal SR, Spence DW, Kato H, Cardinali DP: Melatonin in septic shock: Some recent concepts. J Crit Care 2010, 25:656.

41. Barrett KE, Barman SM, Boitano S, Brooks H: The adrenal medulla and adrenal cortex. In Ganong's Review of Medical Physiology 23'd edition. Edited by Barrett KE, Barman SM, Boitano S, Brooks H. New York: Mc-Graw Hill Companies, 2010:337-362.

42. Kalsbeek A, van der Vliet J, Buijs RM: Decrease of endogenous vasopressin release necessary for expression of the circadian rise in plasma corticosterone: a reverse microdialysis study. J Neuroendocrinol 1996, 8:299-307.

43. Weibel L, Follenius M, Spiegel K, Ehrhart J, Brandenberger G: Comparative effect of night and daytime sleep on the 24-hour cortisol secretory profile Sleep 1995, 18:549-556.

44. Jung $C M$, Khalsa SB, Scheer FA, et al:: Acute effects of bright light exposure on cortisol levels. J Biol Rhythms 2010, 25:208-216.

45. Chrousos GP: The hypothalamic-pituitary-adrenal axis and immunemediated inflammation. N Engl J Med 1995, 332:1351-1362.

46. Jäättelä M, Ilvesmäki V, Voutilainen R, Stenman UH, Saksela E: Tumor necrosis factor as a potent inhibitor of adrenocorticotropin-induced cortisol production and steroidogenic p450 enzyme gene expression in cultured human fetal adrenal cells. Endocrinology 1991, 128:623-629.

47. Annane D, Sebille V, Troche G, Raphael JC, Gajdos P, Bellissant E: A 3-level prognostic classification in septic shock based on cortisol levels and cortisol response to corticotropin. JAMA 2000, 283:1038-1045.

48. Molijn GJ, Koper JW, van Uffelen CJ, et al:: Temperature-induced downregulation of the glucocorticoid receptor in peripheral blood mononuclear leucocyte in patients with sepsis or septic shock. Clin Endocrinol (Oxf) 1995, 43:197-203.

49. Zeitzer JM, Dijk DJ, Kronauer R, Brown E, Czeisler C: Sensitivity of the human circadian pacemaker to nocturnal light: melatonin phase resetting and suppression. J Physiol 2000, 3:695-702.

50. Figueiro MG, Rea MS: The effects of red and blue lights on circadian variations in cortisol, alpha amylase, and melatonin. Int J Endocrinol 2010, 2010:829351.

51. Ishida A, Mutoh T, Ueyama T, et al:: Light activates the adrenal gland: timing 
of gene expression and glucocorticoid release. Cell Metab 2005, 2:297-307.

52. Nelson RJ, Drazen DL: Melatonin mediates seasonal changes in immune function. Ann N Y Acad Sci 2000, 917:404-415.

53. Martin LB, Weil ZM, Nelson RJ: Seasonal changes in vertebrate immune activity: mediation by physiological trade-offs. Philos Trans R Soc Lond B Biol Sci 2008, 363:321 -339.

54. Haldar C, Singh R, Guchhait P: Relationship between the annual rhythms in melatonin and immune system status in the tropical palm squirrel, Funambulus pennanti. Chronobiol Int 2001, 18:61-69.

55. Nelson RJ, Demas GE, Klein SL, Kriegsfeld L: The influence of season, photoperiod and pineal melatonin on immune function. J Pineal Res 1995 , 19:149-165.

56. Blom JM, Gerber JM, Nelson RJ: Day length affects immune cell numbers in deer mice: interactions with age, sex, and prenatal photoperiod. Am J Physiol 1994, 267:R596-R601.

57. Tracey KJ: The inflammatory reflex. Nature 2002, 420:853-859

58. Eskandari F, Sternberg EM: Neural-immune interactions in health and disease. Ann NY Acad Sci 2002, 966:20-27.

59. Borovikova LV, Ivanova S, Zhang M, et al:: Vagus nerve stimulation attenuates the systemic inflammatory response to endotoxin. Nature 2000, 405:458-462.

60. Borovikova LV, Ivanova S, Nardi D, et al: Role of vagus nerve signaling in CNI-1493-mediated suppression of acute inflammation. Auton Neurosci 2000, 85:141-147.

61. Giebelen IA, van Westerloo DJ, LaRosa GJ, de Vos AF, van der Poll T: Local stimulation of alpha7 cholinergic receptors inhibits LPS-induced TNF-alpha release in the mouse lung. Shock 2007, 28:700-703.

62. van Westerloo $D J$ : The vagal immune reflex: a blessing from above. Wien Med Wochenschr 2010, 160:112-117.

63. Rosas-Ballina $\mathrm{M}$, Ochani $\mathrm{M}$, Parrish WR, et al: Splenic nerve is required for cholinergic antiinflammatory pathway control of TNF in endotoxemia. Proc Natl Acad Sci USA 2008, 105:11008-11013.

64. Buiis RM, la Fleur SE, Wortel J, et al:: The suprachiasmatic nucleus balances sympathetic and parasympathetic output to peripheral organs through separate preautonomic neurons. J Comp Neurol 2003, 464:36-48.

65. Loser K, Beissert S: Regulation of cutaneous immunity by the environment: An important role for UV irradiation and vitamin D. Int Immunopharmacol 2009, 9:587-589.

66. Loser K, Mehling A, Loeser S, et al: Epidermal RANKL controls regulatory T-cell numbers via activation of dendritic cells. Nat Med 2006 12:1372-1379.

67. Kamen D, Tangpricha V: Vitamin D and molecular actions on the immune system: modulation of innate and autoimmunity. J Mol Med 2010, 88:441-450.

68. Bucki R, Leszczyñska K, Namiot A, Soko ${ }^{3}$ owski W: Cathelicidin LL-37: A multitask antimicrobial peptide. Arch Immunol Ther Exp (Warsz) 2010, 58:15-25.

69. Zasloff M: Sunlight, vitamin $D$, and the innate immune defenses of the human skin. J Invest Dermatol 2005, 125: 6-17.
70. Jeng L, Yamshchikov AV, Judd SE, et al:: Alterations in vitamin D status and anti-microbial peptide levels in patients in the intensive care unit with sepsis. J Trans/ Med 2009, 7:28.

71. van den Berg A: Health Impacts of Healing Environments. The Architecture of Hospitals. University Hospital Groningen, Groningen; 2005.

72. Ulrich R: View through a window may influence recovery from surgery. Science 1984, 224:420-421.

73. Rubin HR, Owens AJ, Golden G, Status report: An investigation to determine whether the built environment affects patients' medical outcomes. The Center for Health Design, Martinez, CA; 1998.

74. Lewy AJ, Kern HA, Rosenthal NE, Wehr TA: Bright artificial light treatment of a manic-depressive patient with seasonal mood cycle. Am J Psychiatry 1982, 139:1496-1498.

75. Kent ST, McClure LA, Crosson WL, Arnett DK, Wadley VG, Sathiakumar N: Effect of sunlight exposure on cognitive function among depressed and non-depressed participants: a REGARDS cross-sectional study. Environ Health 2009, 8:34

76. Beauchemin KM, Hays P: Dying in the dark: sunshine, gender and outcomes in myocardial infarction. JR Soc Med 1998, 91:352-354.

77. Van Rompaey B, Elseviers MM, Schuurmans MJ, Shortridge-Baggett LM, Truijen S, Bossaert L: Risk factors for delirium in intensive care patients: a prospective cohort study. Crit Care 2009, 13:R77.

78. Heerwagen JH, Heerwagen DR: Lighting and psychological comfort. Lighting Design and Application 1986, 16:47-51.

79. Boyce P, Hunter C, Howlett O: The Benefits Of Daylight Through Windows. Lighting Research Center, New York; 2003.

80. Kiefer RA: An integrative review of the concept of well-being. Holist Nurs Pract 2008, 22: 44-252.

81. Huppert FA, Baylis N: Well-being: towards an integration of psychology, neurobiology and social science. Philos Trans R Soc Lond B Biol Sci 2004, 359:1447-1451.

82. Fredrickson BL: The broaden-and-build theory of positive emotions. Philos Trans R Soc Lond B Biol Sci 2004, 359:1367-1378.

83. Zeidan F, Johnson SK, Gordon NS, Goolkasian P: Effects of brief and sham mindfulness meditation on mood and cardiovascular variables. J Altern Complement Med 2010, 16:867-873.

84. Davidson RJ, Kabat-Zinn J, Schumacher J, et al:: Alterations in brain and immune function produced by mindfulness meditation. Psychosom Med 2003, 65: 64-570

85. Moos RH: The Human Context: Environmental Determinants Of Behavior. Wiley, New York; 1976.

doi:10.1186/cc10000

Cite this article as: Castro $\mathrm{R}$, et al: The effect of light on critical illness. Critical Care 2011, 15:218. 